\title{
La incidencia de los estilos de aprendizaje en el índice de disponibilidad léxica individual de estudiantes extranjeros de español
}

Palabras clave: estilo de aprendizaje, léxico, disponibilidad léxica, léxico disponible, español como lengua extranjera

\section{Introducción}

El presente trabajo pretende determinar si existen relaciones entre los estilos de aprendizaje de los aprendices de español como lengua extranjera y su índice de disponibilidad léxica individual. Aunque ambos temas han sido investigados de manera independiente en el ámbito de la enseñanza de segundas lenguas, nunca han sido abordados conjuntamente con el objetivo de analizar las posibles correlaciones existentes entre ellos. Para identificar los estilos de aprendizaje se han utilizado cinco cuestionarios, que permiten englobar más estilos de los que se pueden abarcar con uno solo. Para el índice de disponibilidad, se han empleado las nociones específicas del Plan curricular del Instituto Cervantes (2006) como centros de interés.

\section{Marco teórico}

\subsection{Los estilos de aprendizaje}

La definición de los estilos de aprendizaje (EA) más utilizada en la bibliografía es la de Keefe (1979: 4): «Learning Style is the composite of characteristic cognitive, affective, and physiological factors that serve as relatively stable indicators of how a learner perceives, interacts with, and responds to the learning environment». Es un constructo controvertido debido a tres motivos: a) 
la existencia de numerosos y diferentes modelos teóricos, b) la falta de homogeneidad terminológica y c) la insuficiencia de datos empíricos que justifiquen la validez de las teorías.

En el primer caso, diversos autores (Curry, 1983; Riding y Rayner, 2000; Coffield, Moseley, Hall y Ecclestone, 2004) han elaborado taxonomías en las que agrupan todos los estilos. La de Coffield et al. (2004), que ha sido muy bien acogida en la comunidad científica, clasifica más de 50 modelos de EA en cinco familias:

a) Factores genéticos y constitutivos: estos estilos se basan en la preferencia por alguno de los sentidos ${ }^{1}$ (Paivio, 1971), en la influencia de la genética (Pederson, Plomin y McClearn, 1994) o en la dominancia de los hemisferios cerebrales (Herrmann, 1982; Dunn, 1990).

b) Estructura cognitiva: son concebidos como la manera habitual en la que un individuo piensa, organiza y representa la información (Riding y Rayner, 2000).

c) Tipo de personalidad: se consideran una parte de la personalidad del individuo (Myers, 1978).

d) Preferencias de aprendizaje: los estilos son una preferencia por una manera de aprender que cambia según el contexto (Honey y Mumford 1986; Kolb², 2000).

e) Estrategias y enfoques de aprendizaje: se tienen en cuenta los efectos de la experiencia previa y el contexto (Entwistle, 1979; Vermunt, 1996).

En cuanto a la diversidad terminológica, expresiones referidas a realidades distintas (estilos cognitivos, estilos de aprendizaje, estrategias de aprendizaje e incluso inteligencias múltiples) se han considerado sinónimas. Esto ha impedido contar con definiciones homogéneas de los conceptos clave, como indica Cassidy (2004: 420): «Defining the key terms in this area is not a straightforward task. The terms learning style, cognitive style and learning strategy are - understandably - used imprecisely in theoretical and empirical accounts in the topic».

Por último, sobre la validez de las teorías, Coffield et al. (2004) comprobaron que casi ninguno de los modelos de EA que analizaron reunía los criterios mínimos de rigor científico: consistencia interna, fiabilidad, validez de

1 En esta corriente se incluye el modelo VAKT, que es el más difundido y engloba todas las propuestas teóricas que proponen los estilos visual, auditivo, cinestésico y táctil.

Este es el autor de esta tradición más influyente en Europa. 
constructo y validez predictiva. Asimismo, Pashler et al. (2008) también concluyen que los trabajos sobre los EA no cuentan con la validez necesaria

A pesar de estas limitaciones, los EA gozan de una amplia aceptación entre los profesores de español como lengua extranjera (Domínguez, López y Andión, 2019), aunque algunos autores consideren que son un mito educativo (Geake, 2008; Howard-Jones, 2014; Newton, 2015) o incluso lleguen a cuestionar su existencia (Willingham, Hughes y Dobolyi, 2015).

\subsection{La disponibilidad léxica}

El concepto de disponibilidad léxica (DL) surge en Francia a mediados del siglo $\mathrm{XX}$ durante el proceso de elaboración de un manual de enseñanza de francés pensado para los inmigrantes que llegaban a Francia. Para establecer el léxico que debían adquirir, los autores (Gougenheim, Michéa, Rivenc y Sauvageot, 1956) elaboraron listas de frecuencia de palabras, pero observaron que muchos términos que eran habituales en las conversaciones cotidianas no aparecían en los listados. Se dieron cuenta de que tales expresiones estaban supeditadas al tema del texto.

Ante esta situación, Gougenheim et al. (1956) utilizaron una nueva metodología, que consistía en pedir a los informantes que proporcionaran las veinte primeras palabras que se les vinieran a la mente sobre dieciséis campos semánticos, que denominaron centros de interés ${ }^{3}$ (CI). La frecuencia no fue el único elemento que tuvieron en cuenta para calcular la disponibilidad, sino también la posición que ocupaban las palabras en la lista de los participantes, de manera que se consideraban más disponibles las que estuvieran en los primeros puestos.

Las investigaciones de DL sobre el español como lengua extranjera ${ }^{4}$ cuentan con dos líneas principales de investigación: una se lleva a cabo en países de habla no hispana (Šifrar Kalan, 2009; Magnúsdóttir, 2012; Rubio Lastra, 2018, entre otros) y la otra se desarrolla en países hispanohablantes, en los que los participantes tienen distintas lenguas maternas (Samper Hernández, 2002; Sánchez-Saus, 2010; Jiménez Berrio, 2013). También existen trabajos sobre otros temas, como la disponibilidad léxica de los manuales (Benítez Pérez, 1997), el léxico disponible en hablantes monolingües y bilingües (Pearson,

3 Sánchez-Saus (2011, pp. 105-108) indica que Gougenheim et al. (1956) adoptaron la expresión centro de interés del ámbito de la pedagogía, en el que Decroly fue su ideólogo, aunque dicho concepto existía con anterioridad en el campo de la lexicografía.

Para una visión detallada sobre el tema, consúltese López González (2014). 
Fernández y Oller, 1993), las relaciones entre el léxico disponible del español como lengua materna y lengua extranjera (Šifrar Kalan, 2012) o varias propuestas de CI específicos para ELE (Bartol Hernández, 2010; Sánchez-Saus, 2011; Paredes García, 2014).

\section{Metodología}

La investigación realizada es cuasiexperimental (porque la muestra no se ha seleccionado de forma aleatoria), transversal (porque no hemos realizado varias mediciones a lo largo del tiempo), correlacional (porque pretendemos medir el grado de relación que hay entra los EA y la DL), bivariada (porque solo hemos considerado dos variables: EA y DL) y exploratoria (porque no hay ningún estudio previo parecido).

\subsection{Descripción de la investigación}

El trabajo de campo lo hemos realizado en dos sesiones. En la primera, las informantes han cumplimentado el cuestionario sociodemográfico y los cinco cuestionarios sobre los EA. En la segunda sesión han hecho la prueba de disponibilidad, en la que hemos seguido este procedimiento: hemos repartido a cada participante una hoja de respuestas y hemos proyectado el primer CI durante 90 segundos para que escribieran las palabras. Después, hemos recogido las hojas de respuestas y hemos repartido las del siguiente CI. Hemos procedido igual con todos los CI.

\subsection{Hipótesis y objetivos de la investigación}

La hipótesis de nuestra investigación, que a su vez se convierte en el objetivo general de la misma, es determinar si existe algún tipo de relación entre los EA y la DL.

\subsection{La población y muestra del estudio}

La muestra se compone de 27 estudiantes de la Facultad de Filosofía y Letras de la Universidad de Ljubljana (Eslovenia). Todos los informantes tienen el mismo género (mujeres), la misma lengua materna (esloveno), el mismo nivel de español (B1), y cursan los mismos estudios universitarios (primero de Lengua y literatura españolas). En la tabla 1 se pueden observar las características de la muestra. 


\begin{tabular}{|c|c|c|c|c|c|}
\hline \multirow{2}{*}{ Informantes } & \multirow{2}{*}{ Edad } & \multirow{2}{*}{$\begin{array}{c}\text { Años de } \\
\text { aprendizaje }\end{array}$} & \multicolumn{2}{|c|}{ Estancias } & \multirow{2}{*}{ Otras lenguas } \\
\hline & & & Lugar & Duración & \\
\hline 1 & 19 & 5 & España & 2 semanas & Inglés \\
\hline 2 & 19 & 6 & - & - & Inglés, ruso y croata \\
\hline 3 & 19 & 5 & España & 1 semana & Inglés \\
\hline 4 & 20 & 2 & España & 1 año & Inglés y alemán \\
\hline 5 & 19 & 4 & España & 1 mes & Inglés \\
\hline 6 & 19 & 8 & España & 3 semanas & Inglés \\
\hline 7 & 20 & 2 & España & 1 año & Inglés \\
\hline 8 & 19 & 3 & - & - & Inglés y francés \\
\hline 9 & 22 & 5 & - & - & Inglés y croata \\
\hline 10 & 19 & 5 & $\begin{array}{c}\text { Argentina } \\
\text { España }\end{array}$ & $\begin{array}{c}1 \text { mes } \\
1 \text { semana }\end{array}$ & Inglés \\
\hline 11 & 20 & 7 & España & 1 semana & Inglés y alemán \\
\hline 12 & 19 & 5 & - & - & Inglés, serbio e italiano \\
\hline 13 & 20 & 8 & España & 5 meses & Inglés, francés y croata \\
\hline 14 & 21 & 5 & España & 1 semana & Inglés, italiano y croata \\
\hline 15 & 19 & 7 & España & 1 semana & Inglés, ruso y serbio \\
\hline 16 & 19 & 7 & España & 3 semanas & Inglés y francés \\
\hline 17 & 19 & 4 & - & - & Inglés, italiano y francés \\
\hline 18 & 19 & 8 & España & 1 semana & Inglés y ruso \\
\hline 19 & 20 & 3 & - & - & Inglés, francés y portugués \\
\hline 20 & 19 & 5 & España & 1 semana & Inglés, serbio y croata \\
\hline 21 & 19 & 5 & España & 1 mes & $\begin{array}{l}\text { Inglés, ruso, alemán, } \\
\text { portugués y croata }\end{array}$ \\
\hline 22 & 20 & 5 & España & 2 semanas & Inglés \\
\hline 23 & 19 & 5 & España & 1 mes & Inglés y croata \\
\hline 24 & 19 & 4 & España & 2 semanas & Inglés \\
\hline 25 & 19 & 3 & España & 1 semana & Inglés, alemán y croata \\
\hline 26 & 24 & 3 & $\begin{array}{c}\text { España } \\
\text { Uruguay } \\
\text { Argentina }\end{array}$ & $\begin{array}{c}1 \text { mes } \\
2 \text { semanas } \\
2 \text { semanas }\end{array}$ & Inglés \\
\hline 27 & 21 & 5 & - & - & Inglés y alemán \\
\hline
\end{tabular}

Tabla 1: Perfil sociodemográfico de la muestra de la investigación

\subsection{Instrumentos}

En esta sección expondremos los cuestionarios para identificar los EA y los recursos de la prueba de DL. Aparte de estos instrumentos, también hemos empleado un cuestionario sociodemográfico (cuyo contenido se desprende de lo expuesto en la tabla 1), así como el programa informático SPSS para el análisis estadístico de los datos. 


\subsubsection{Los cuestionarios sobre los estilos de aprendizaje}

Aunque lo habitual es utilizar un único instrumento sobre los EA, en nuestro estudio hemos empleado cinco (tabla 2) por dos motivos. En primer lugar, porque ningún cuestionario mide todas las dimensiones que están implicadas desde el punto de vista teórico. Y, en segundo lugar, porque hay muy pocos instrumentos válidos y fiables, como indicaron Coffield et al., (2004), de manera que si se utiliza más de uno se obtendrían más evidencias sobre la validez y fiabilidad de los cuestionarios. Un último criterio que hemos tenido en cuenta ha sido que estuvieran escritos en inglés, ya que todas las informantes poseen, al menos, un nivel $\mathrm{B}_{2}$ en esta lengua y los pueden entender sin dificultad, con lo que evitamos los problemas de validación que conlleva la traducción.

\begin{tabular}{|c|c|}
\hline Cuestionarios & Estilos \\
\hline $\begin{array}{l}\text { Perceptual Learning Style Preference Questionnaire (PLSPQ) } \\
\text { Reid (1995) }\end{array}$ & $\begin{array}{l}\text { Visual } \\
\text { Auditivo } \\
\text { Táctil } \\
\text { Cinestésico } \\
\text { Grupal } \\
\text { Individual }\end{array}$ \\
\hline $\begin{array}{l}\text { Inventory of Learning Styles in Higher Education (ILSHE) } \\
\text { Vermunt (1994) }\end{array}$ & \begin{tabular}{|l|} 
Significativo \\
Repetitivo \\
Práctico \\
No dirigido \\
\end{tabular} \\
\hline $\begin{array}{l}\text { Cognitive Style Index (CSI) } \\
\text { Allinson y Hayes (1996) }\end{array}$ & $\begin{array}{l}\text { Intuitivo } \\
\text { Quasi-intuitivo } \\
\text { Adaptativo } \\
\text { Quasi-analista } \\
\text { Analista } \\
\end{array}$ \\
\hline $\begin{array}{l}\text { Approaches and Study Skills Inventory for Students (ASSIST) } \\
\text { Entwistle (1998) }\end{array}$ & $\begin{array}{l}\text { Profundo } \\
\text { Estratégico } \\
\text { Superficial } \\
\end{array}$ \\
\hline $\begin{array}{l}\text { Learning Style Survey (LSS) } \\
\text { Cohen, Oxford y Chi (2002) }\end{array}$ & $\begin{array}{l}\text { Visual/auditivo/cinestésico } \\
\text { Extrovertido/introvertido } \\
\text { Aleatorio/concreto } \\
\text { Cierre/abierto } \\
\text { Global/particular } \\
\text { Sintético/analítico } \\
\text { Detallista/generalista } \\
\text { Deductivo/inductivo } \\
\text { Independiente/dependiente } \\
\text { Impulsivo/reflexivo } \\
\text { Metafórico/literal }\end{array}$ \\
\hline
\end{tabular}

Tabla 2: Cuestionarios para identificar los EA utilizados en la investigación 


\subsubsection{Los centros de interés}

Basándonos en Paredes García (2014), hemos seleccionado seis nociones específicas de entre las veinte del PCIC (2006): 1) Individuo: dimensión física, 2) Individuo: dimensión perceptiva y anímica, 3) Salud e higiene, 4) Ocio, 5) Geografía y naturaleza y 6) Viajes, alojamiento y transportes.

\subsubsection{Las proyecciones de los centros de interés}

Se ha elaborado una presentación en PowerPoint, constituida por una diapositiva para cada CI (anexo 1) en la que aparece el nombre del centro junto con las subcategorías con las que viene acompañado en el PCIC (2006). Las subcategorías funcionan como estímulos facilitadores de la búsqueda léxica. Cada diapositiva se ha proyectado durante 90 segundos.

\subsubsection{Las plantillas de respuesta}

En vez de hojas en blanco se han utilizado plantillas de respuesta (anexo 2) para garantizar la validez interna de la prueba. Por una parte, conseguimos que todas las participantes escriban las palabras en el mismo orden, ya que cuando hemos utilizado folios en blanco en otras ocasiones, algunas personas escribían de izquierda a derecha y otras de arriba abajo, con lo cual el orden de las palabras era diferente en cada caso. Por otro lado, hemos elaborado plantillas para cada centro para repartirlas y recogerlas después del tiempo de respuesta y así evitar que añadan palabras fuera del tiempo establecido.

\subsubsection{Los criterios de edición}

Para homogeneizar las diferentes formas de escribir un mismo vocablo por parte de las informantes, hemos seguido los criterios de edición de Samper Padilla (1998), que son comunes a todos los trabajos de disponibilidad, y los de Samper Hernández (2002), específicos para las investigaciones sobre el español como lengua extranjera. Como norma de corrección lingüística nos hemos basado en los criterios de la RAE.

\section{Resultados}

En esta sección exponemos los siguientes resultados sobre la muestra: las posibles correlaciones entre las variables sociodemográficas y la DL, sus EA, 
su producción léxica, el índice de disponibilidad léxica individual (IDI) y las posibles correlaciones entre sus EA y su DL, que es el aspecto central de nuestro estudio.

\subsection{Los rasgos sociodemográficos de la muestra y su disponibilidad léxica}

Como se muestra en la tabla 3, no hay correlaciones estadísticamente significativas entre los rasgos sociodemográficos de la muestra y la DL. Esto es en sí mismo una información muy ilustrativa sobre el funcionamiento de la disponibilidad, pues se nos presenta como un constructo ajeno a estos rasgos. Otra posible explicación podría consistir en la homogeneidad de la muestra, de modo que las diferencias existentes en cada variable no son tan relevantes como para propiciar cambios significativos.

\begin{tabular}{|l|c|c|c|c|}
\cline { 2 - 5 } \multicolumn{1}{c|}{} & Edad & Idiomas & $\begin{array}{c}\text { Tiempo } \\
\text { de aprendizaje }\end{array}$ & Estancias \\
\hline $\begin{array}{l}\text { Correlación } \\
\text { de Pearson }\end{array}$ & 0,042 & 0,015 & 0,102 & $-0,288$ \\
\hline Sig. (bilateral) & 0,834 & 0,940 & 0,611 & 0,146 \\
\hline
\end{tabular}

Tabla 3: Matriz de correlaciones de los rasgos sociodemográficos y la DL de la muestra

\subsection{Los estilos de aprendizaje de la muestra}

En la tabla 4 mostramos los estadísticos descriptivos de la muestra para cada uno de los cuestionarios. Los estilos de aprendizaje con mayor puntuación se pueden considerar los preferentes. Puesto que cada instrumento aborda diferentes dimensiones, la suma de todas ellas nos permite tener una visión global del perfil de aprendizaje de las estudiantes. 


\begin{tabular}{|c|c|c|c|c|c|}
\hline \multirow{2}{*}{ Cuestionarios } & \multirow{2}{*}{ Estilos } & \multicolumn{4}{|c|}{ ESTADÍSTICOS DESCRIPTIVOS } \\
\hline & & Mínimo & Máximo & Media & Desviación \\
\hline \multirow{3}{*}{ ASSIST } & Estratégico & 11 & 26 & 18,9 & 4,29 \\
\hline & Profundo & 14 & 29 & 21,4 & 3,32 \\
\hline & Superficial & 11 & 24 & 17,4 & 3,46 \\
\hline \multirow{5}{*}{ CSI } & Intuitivo & - & $22 \%$ & - & - \\
\hline & Cuasi-intuitivo & - & $48 \%$ & - & - \\
\hline & Adaptativo & - & $19 \%$ & - & - \\
\hline & Cuasi-analítico & - & $11 \%$ & - & - \\
\hline & Analítico & - & $0 \%$ & - & - \\
\hline \multirow{4}{*}{ ILSHE } & Significativo & 2,1 & 4,5 & 3,1 & 0,53 \\
\hline & Repetitivo & 2,3 & 4,6 & 3,3 & 0,5 \\
\hline & Práctico & 2,6 & 4,8 & 3,6 & 0,47 \\
\hline & No dirigido & 2 & 3,9 & 3 & 0,41 \\
\hline \multirow{19}{*}{ LSS } & Visual & 19 & 32 & 26,4 & 3,55 \\
\hline & Auditivo & 15 & 30 & 21,8 & 4,61 \\
\hline & Cinestésico/táctil & 9 & 32 & 20,2 & 6,11 \\
\hline & Extrovertido & 8 & 22 & 13,5 & 3,58 \\
\hline & \begin{tabular}{|l|} 
Introvertido \\
\end{tabular} & 5 & 22 & 13,5 & 4,19 \\
\hline & Intuitivo & 11 & 20 & 15,2 & 2,63 \\
\hline & Secuencial & 9 & 19 & 13,3 & 2,99 \\
\hline & Global & 5 & 17 & 11,3 & 2,36 \\
\hline & Particular & 7 & 17 & 11,0 & 2,23 \\
\hline & Sintético & 10 & 17 & 13 & 1,84 \\
\hline & Analítico & 4 & 14 & 9,5 & 2,47 \\
\hline & \begin{tabular}{|l|} 
Agudizador \\
\end{tabular} & 4 & 12 & 7,3 & 2 \\
\hline & Nivelador & 2 & 11 & 6,6 & 2,1 \\
\hline & Deductivo & 4 & 12 & 8,4 & 2,24 \\
\hline & Inductivo & 4 & 10 & 6,4 & 1,62 \\
\hline & Independiente & 4 & 11 & 8 & 1,9 \\
\hline & Dependiente & 3 & 10 & 6,9 & 2,17 \\
\hline & Impulsivo & 3 & 10 & 6,9 & 2,17 \\
\hline & Reflexivo & 4 & 12 & 7,3 & 1,98 \\
\hline \multirow{6}{*}{ PLSPQ } & Visual & 22 & 44 & 33,8 & 5,85 \\
\hline & Auditivo & 22 & 48 & 35,8 & 5,75 \\
\hline & Táctil & 16 & 44 & 32,1 & 6,72 \\
\hline & Cinestésico & 26 & 48 & 35,3 & 5,38 \\
\hline & Grupal & 10 & 42 & 27,7 & 8,53 \\
\hline & Individual & 20 & 50 & 35,6 & 7,71 \\
\hline
\end{tabular}

Tabla 4: Estadísticos descriptivos de los EA de la muestra 
En primer lugar, según el ASSIST, la muestra manifiesta una tendencia preferente hacia el estilo profundo, lo cual se traduce en que el objetivo de aprendizaje de las alumnas es comprender los contenidos lingüísticos. Para ello se involucran en el curso como consecuencia de su motivación intrínseca y se basan en sus experiencias previas para aprender los conceptos nuevos.

Según el CSI, gran parte de las estudiantes son cuasi-intuitivas, lo que significa que en vez de confiar plenamente en su intuición, la ponen en entredicho y buscan información en otras fuentes cuando tienen que tomar alguna decisión. Sin embargo, esto contrasta con los datos proporcionados por el LSS, en el que el estilo preferido es el intuitivo.

El estilo predominante según el ILSHE es el práctico, caracterizado por una concepción sobre el aprendizaje que pone el énfasis en la utilidad del mismo, cuyas estrategias de procesamiento son concretas y con un interés por aprender tanto por vocación como orientado a la obtención de un título. Esto último no coincidiría con el estilo profundo del ASSIST, caracterizado por una motivación intrínseca.

En cuanto a las preferencias estilísticas que nos proporcionan el LSS y el PLS$\mathrm{PQ}$, observamos contradicciones en los resultados. Así, y por lo que respecta a la dimensión perceptiva, según el primero la muestra sería preferentemente visual, mientras que, según el segundo, sería auditiva. También resulta contradictorio el estilo extrovertido del LSS frente al individual del PLSPQ, ya que el primero se caracteriza por preferir actividades que requieran interacción social mientras que el principal rasgo definitorio del segundo es su preferencia por trabajar a solas.

De los demás estilos del LSS, no existe una preferencia claramente definida entre los estilos global y particular, lo cual implicaría que las alumnas son igual de buenas captando la idea principal de un texto, aunque no conozcan todo el vocabulario (global), como recordando información específica sobre un tema (particular). Estos dos estilos, a su vez, conectan con otros del mismo cuestionario. Así, el estilo global encaja bien con el sintético, caracterizado por la capacidad de resumir bien, y el particular, con el agudizador, que es capaz de distinguir las diferencias que hay entre elementos similares, lo cual también tiene vínculos con el independiente de campo, que puede extraer de un contexto la información requerida incluso en presencia de elementos distractores. Por otro lado, el estilo deductivo indica que las alumnas prefieren comenzar por las reglas generales en vez de con ejemplos y, por último, el estilo reflexivo hace que las estudiantes se tomen su tiempo antes de responder. 


\subsection{La producción léxica de la muestra del estudio}

En este apartado presentamos los datos sobre las lexías 5 proporcionadas por las informantes en las listas de disponibilidad. Como se muestra en la tabla 5, hay notables diferencias en la productividad de las palabras, pues oscila entre las 275 del CI 2 y las 444 del CI 1. Esta amplia horquilla se reduce en el caso de los vocablos, como se desprende de una menor desviación estándar; aquí, el CI 3 presenta el valor mínimo (134) y el CI 5, el máximo (181). Por último, la media de vocablos por centro también es variada, pues se sitúa entre los 10,2 del CI 2 y los 16,4 del CI 1. En síntesis, el más productivo tanto en palabras como en media de vocablos es el CI 1 (Individuo: dimensión física), mientras que en el extremo opuesto se sitúa el CI 2 (Individuo: dimensión perceptiva). Nos resulta paradójico que estos dos centros de interés, tan estrechamente relacionados al referirse a dos dimensiones del individuo (la física y la perceptiva), sean los que presenten el rendimiento más opuesto.

\begin{tabular}{|l|c|c|c|c|c|c|c|c|}
\cline { 2 - 8 } \multicolumn{1}{c|}{} & Cl 1 & CI 2 & CI 3 & CI 4 & CI 5 & Cl 6 & Media & Desviación \\
\hline Palabras & 444 & 275 & 299 & 384 & 392 & 417 & 368,5 & 66,95 \\
\hline Vocablos & 153 & 170 & 134 & 153 & 181 & 166 & 159,5 & 16,42 \\
\hline Media de vocablos & 16,4 & 10,2 & 11,1 & 14,2 & 14,5 & 15,4 & 13,6 & 2,45 \\
\hline
\end{tabular}

Tabla 5: Estadísticos descriptivos de la producción léxica por centros de interés

\subsection{La disponibilidad léxica de la muestra del estudio}

Debido a las características de nuestra investigación, que pretende determinar si existen relaciones entre los EA de las participantes y su DL, hemos calculado el índice de disponibilidad léxica individual (IDI) de cada informante para cada centro de interés, así como el índice global, que es el resultado de la suma de todos los anteriores (ver anexo 3). Para ello, hemos aplicado la fórmula de López Chávez y Strassburger Frías (1991).

\subsection{Relaciones entre los estilos de aprendizaje y la disponibilidad léxica}

Para determinar si existen relaciones entre los EA y la DL, hemos calculado el coeficiente de correlación de Pearson entre los resultados de los cuestionarios

5 Como viene siendo habitual en los trabajos sobre disponibilidad, utilizamos el término vocablo para referirnos a las unidades léxicas sin considerar las veces que se repite, y palabra para incluir las repeticiones. 
sobre los EA y el IDI de las participantes. Hemos utilizado esta prueba no paramétrica porque hemos trabajado con una única muestra, que además es muy pequeña $(\mathrm{N}=27)$, y por el carácter de las variables, que son categóricas. Los resultados (tabla 6) indican que solo hay correlaciones estadísticamente significativas en cuatro estilos (práctico, grupal, visual y global), correspondientes a tres cuestionarios (ILSHE, PLSPQy LSS).

\begin{tabular}{|l|c|c|c|c|}
\hline ASSIST & Estratégico & Profundo & Superficial & CSI \\
\hline $\begin{array}{l}\text { Correlación } \\
\text { de Pearson }\end{array}$ & 0,212 & 0,144 & $-0,192$ & 0,025 \\
\hline Sig. (bilateral) & 0,288 & 0,473 & 0,338 & 0,901 \\
\hline
\end{tabular}

\begin{tabular}{|l|c|c|c|c|}
\hline ILSHE & Significativo & Repetitivo & Práctico & No dirigido \\
\hline $\begin{array}{l}\text { Correlación } \\
\text { de Pearson }\end{array}$ & 0,238 & 0,310 &, $\mathbf{4 0 2}^{*}$ & $-0,094$ \\
\hline Sig. (bilateral) & 0,233 & 0,115 & 0,038 & 0,640 \\
\hline
\end{tabular}

\begin{tabular}{|l|c|c|c|c|c|c|}
\hline PLSPQ & Visual & Auditivo & Táctil & Cinestésico & Grupal & Individual \\
\hline $\begin{array}{l}\text { Correlación } \\
\text { de Pearson }\end{array}$ & $-0,079$ & 0,148 & 0,234 & 0,198 & $\mathbf{4 4 6}$ & $-0,262$ \\
\hline Sig. (bilateral) & 0,695 & 0,460 & 0,241 & 0,321 & 0,020 & 0,187 \\
\hline
\end{tabular}

\begin{tabular}{|l|c|c|c|c|c|c|c|c|c|}
\hline LSS & Visual & Aud. & Tác/Cin. & Extr. & Intr. & Intu. & Secu. & Glob. & Part. \\
\hline $\begin{array}{l}\text { Correlación } \\
\text { de Pearson }\end{array}$ & $\mathbf{, 5 1 5 *}^{* *}$ & $-0,085$ & 0,358 & 0,157 & 0,124 & 0,233 & 0,015 &, $\mathbf{4 3 7}^{*}$ & $-0,063$ \\
\hline Sig. (bilateral) & 0,006 & 0,672 & 0,067 & 0,433 & 0,538 & 0,242 & 0,942 & 0,023 & 0,756 \\
\hline
\end{tabular}

\begin{tabular}{|l|c|c|c|c|c|c|c|c|c|c|}
\hline LSS & Sintét. & Analít. & Agud. & Nivel. & Deduc. & Induc. & Ind. & Dep. & Imp. & Refl. \\
\hline $\begin{array}{l}\text { Correlación } \\
\text { de Pearson }\end{array}$ & 0,297 & 0,208 & 0,176 & 0,166 & $-0,009$ & 0,152 & 0,185 & 0,189 & 0,189 & 0,233 \\
\hline Sig. (bilateral) & 0,133 & 0,299 & 0,379 & 0,408 & 0,966 & 0,449 & 0,356 & 0,346 & 0,346 & 0,242 \\
\hline
\end{tabular}

Tabla 6: Matriz de correlaciones entre los estilos de aprendizaje y la disponibilidad léxica

Conviene hacer un análisis más riguroso sobre estos datos. Así, tanto el PLSPQ como el LSS incluyen el estilo visual, pero solo en el segundo caso se produce una correlación significativa. Además, el signo de la correlación en ambos cuestionarios es distinta: negativo en el PLSPQy positivo en el LSS. Lo mismo ocurre con el estilo auditivo que, aunque no presenta correlación significativa en ninguno de los dos cuestionarios, su signo es opuesto en cada caso. Un hecho similar sucede con el estilo grupal del PLSPQ, que es parecido al extrovertido del LSS, pero mientras que el primero sí presenta correlación significativa, el segundo, no. Si a esto añadimos que el estilo práctico del ILSHE y el global del LSS no se parecen a otros, podemos concluir que son los únicos que correlacionan de manera fiable con la DL. 
Si observamos el signo de las correlaciones estadísticamente significativas, la incidencia de los cuatro estilos de aprendizaje (práctico, grupal, visual y global) sobre la DL es positiva. Esto significa que unas puntuaciones elevadas en dichos estilos llevan aparejadas unos altos índices de DL individual. No obstante, no podemos concluir que se trata de relaciones de causalidad, es decir, que tales estilos causan una alta disponibilidad, ya que el índice de correlación solo indica relación, pero no causalidad, que habría que buscarla en otro lugar.

\section{Conclusiones}

En primer lugar, el hecho de que solo haya correlaciones estadísticamente significativas con cuatro estilos indica que la incidencia de estos sobre la DL es mínima. Si a esto añadimos que de los cuatro estilos, dos de ellos (el grupal y el visual) presentan resultados distintos según el cuestionario que se aplique (el LSPQ o el LSS), solo se pueden considerar correlaciones fiables las de los estilos práctico y global. Por tanto, los estilos no son una variable que influye de manera significativa en la DL. Esto implica que la disponibilidad es, en general, independiente de los EA y que cualquier persona, con independencia de su estilo (excepto el práctico y el global), puede alcanzar un alto grado de DL.

De lo anterior se desprende que ha sido una decisión metodológica acertada utilizar más de un cuestionario, ya que si solo hubiéramos empleado uno y hubiese sido el ASSIST o el CSI, habríamos concluido que no hay relaciones entre estilos y disponibilidad. Asimismo, haber utilizado dos cuestionarios (el PLSPQ y el LSS) con estilos similares (auditivo, visual, grupal y extrovertido) nos ha permitido contrastar los resultados, en cuyo caso hemos observado comportamientos opuestos.

No obstante, estas divergencias también podrían deberse a alguna limitación de la investigación, que habría que subsanar en futuros estudios. Por un lado, sugerimos trabajar con una muestra más grande para obtener un perfil más variado de estilos que permita conseguir más correlaciones con la disponibilidad. Por otra parte, sería conveniente contrastar de nuevo los estilos visual, grupal y extrovertido del PLSPQ y del LSS para comprobar si tienen un comportamiento tan divergente como en nuestro estudio. Por último, sería interesante utilizar solo el ASSIST y el CSI para determinar si se produce alguna correlación significativa; en caso negativo, los estilos de estos cuestionarios se podrían descartar como variables que inciden en la DL. 
Finalmente, consideramos que hemos aportado una modesta contribución sobre los EA en el ámbito de la adquisición de lenguas, aunque sea en un sentido contrario al esperado, pues no son un factor influyente; más bien al contrario, su impacto es mínimo, al menos en lo que se refiere a la DL. Teniendo en cuenta la gran aceptación que tiene el constructo de los estilos en el ámbito formativo, es probable que nuestras conclusiones no sean bien recibidas. No obstante, se trata de una investigación de carácter exploratorio, que conviene ampliar con las orientaciones indicadas previamente para poder concluir definitivamente si los EA inciden o no de alguna forma en la DL.

\section{Bibliografía}

Allinson, C. W. y Hayes, J. (1996): «The cognitive style index: A measure of intuition-analysis for organizational research». Journal of Management studies, $33(1), 119-135$.

Bartol Hernández, J. A. (2010): «Disponibilidad léxica y selección del vocabulario». En: Rosa M. ${ }^{a}$ Castañer Martín, Vicente Lagüéns Gracia (eds.), De moneda nunca usada. Estudios dedicados a José M. ${ }^{a}$ Enguita Utrilla. Zaragoza: Institución Fernando el Católico, 85-107.

Benítez Pérez, P. (1997): «El vocabulario enseñado en los manuales de ELE ¿es el adecuado?» En: Alberto Barrera, Vidal (ed.), Problemas actuales en la enseñanza del español como lengua extranjera: gramática, pragmática, vocabulario y cultura. Utrecht: Wolters, 64-76.

Cassidy, S. (2004): «Learning Styles: An overview of theories, models, and measures». Educational Psychology, 24, 419-444.

Coffield, F., Moseley, D., Hall, E., Ecclestone, K. (2004): Learning styles and pedagogy in post-16 learning. A systematic and critical review. Londres: Learning and Skills Research Centre.

Cohen, A., Oxford, R., Chi, J. (2002): Learning Style Survey. Minneapolis: University of Minnesota.

Curry, L. (1983): «An organization of learning styles theory and constructs». En: Lynn Curry (ed.), Learning style in continuing medical education. Ottawa: Canadian Medical Association, 115-123.

Domínguez Pelegrín, J., López Quero, S., Andión Herrero, M. A. (2019): «Las creencias de los profesores de español como lengua extranjera sobre los estilos de aprendizaje». Revista española de lingüística aplicada, 32(2), 419-454. 
Dunn, R. (1990): «Understanding the Dunn and Dunn learning styles model and the need for individual diagnosis and prescription». Reading, Writing and Learning Disabilities, 6, 223-247.

Entwistle, N. J. (1979): «Approaches to learning and levels of understanding». British Educational Research Journal, 1, 5-44.

Entwistle, N. J. (1998). Styles of Learning and Teaching: An Integrated Outline of Educational Psychology for Student Teachers and Lecturers. Londres: David Fulton Publishers.

Geake, J. (2008): «Neuromythologies in education». Educational Research, 50, 123-133.

Gougenheim, G., Michéa, R., Rivenc, P., Sauvageot, A. (1956): L'élaboration du français élémentaire. Paris: Didier.

Herrmann, N. (1982): Herman Brain Dominance Instrument. Lake Lure: Applied Services.

Honey, P., Mumford, A. (1986): The Manual of Learning Styles. Londres: Peter Honey Publications.

Howard-Jones, P. (2014): «Neuroscience and education: Myths and messages». Nature Reviews Neuroscience, 15, 817-824.

Instituto Cervantes (2006): Plan curricular del Instituto Cervantes. Madrid: Instituto Cervantes.

Instituto Cervantes (2018): El español: una lengua viva. Informe 2018: https:// cvc.cervantes.es/lengua/espanol_lengua_viva/pdf/espanol_lengua_ viva_2018.pdf (18-11-2019).

Jiménez Berrio, F. (2013): Léxico disponible de inmigrantes escolares no bispanobablantes. Pamplona: Universidad de Navarra.

Keefe, J. W. (1979): «Learning Style: An Overview». Student Learning Styles: Diagnosing and Prescribing Programs. Reston: NASSP.

Kolb, D. A. (2000): Facilitator's guide to learning. Boston: Hay/McBer.

López Chávez, J., Strassburger Frías, C. (1991): «Un modelo para el cálculo del índice de disponibilidad léxica individual». En: Humberto López Morales (ed.), La enseñanza del español como lengua materna. Río Piedras: Universidad de Puerto Rico, 91-112.

López González, A. M. (2014): «Desarrollo de los estudios de disponibilidad léxica en español lengua extranjera (ELE)». En: Narciso $M$. Contreras Izquierdo (ed.), La enseñanza del Español como LE/L2 en el siglo XXI. Málaga: ASELE, 397-408. 
Magnúsdóttir, S. (2012): Disponibilidad léxica en alumnos de español como lengua extranjera. Estudio sobre el léxico disponible en alumnos de ELE en la secundaria en Islandia. Memoria de licenciatura. Universidad de Islandia.

Myers, I. (1978): Myers-Briggs Type Indicator. Palo Alto: Consulting Psychologists' Press.

Newton, P. M. (2015): «The Learning Styles Myth is Thriving in Higher Education». Frontiers in Psychology, 6, 1908.

Paivio, A. (1971): «Styles and strategies of learning». British Journal of Educational Psychology, 46, 128-148.

Paredes García, F. (2014): «A vueltas con la selección de 'centros de interés' en los estudios de disponibilidad léxica: para una propuesta renovadora a propósito de la disponibilidad léxica en ELE». Revista Nebrija de Linguiistica Aplicada, 16.

Pashler, H., McDaniel, M., Rohrer, D., Bjork, R. (2008): «Learning Styles. Concepts and Evidence». Psychological science in the public interest, 9(3), 105-119.

Pearson, B. Z., Fernández, S., Oller, D. K. (1993): «Lexical development in bilingual infants and toddlers: Comparison to monolingual norms». Language Learning, 43, 93-120.

Pederson, N. L., Plomin, R., McClearn, G. E. (1994): «Is there a G beyond g? (Is there genetic influence on specific cognitive abilities independent of genetic influence on general cognitive ability?)». Intelligence, 18, 133-143.

Reid, J. (1995): Learning Styles in the ESL/EFL Classroom. Boston: Heinle and Heinle.

Riding, R. J., Rayner, S. (2000): Cognitive styles. Conneticut: Ablex Publishing Corporation.

Rubio Lastra, M. (2018): «Disponibilidad léxica de 52 estudiantes taiwaneses universitarios de ELE A1». Marcoele. Revista de didáctica español lengua extranjera, 27.

Samper Hernández, M. (2002): Disponibilidad léxica en alumnos de español como lengua extranjera. Málaga: ASELE.

Samper Padilla, A. (1998): «Criterios de edición del léxico disponible». Lingüistica, 10, 311-333.

Sánchez-Saus Laserna, M. (2010): «La variación en el léxico disponible de los estudiantes de español como lengua extranjera. Resultados cuantitativos». En: Jorge Luis Bueno Alonso (ed.), Analizar datos: Describir variación/ Analysing data: Describing variation. Vigo: Universidad de Vigo, 265-273. 
Sánchez-Saus Laserna, M. (2011): Bases semánticas para el estudio de los centros de interés del léxico disponible. Disponibilidad léxica de informantes extranjeros en las universidades andaluzas. Tesis doctoral inédita. Cádiz: Universidad de Cádiz.

Šifrar Kalan, M. (2009): «Disponibilidad léxica en español como lengua extranjera. El cotejo de las investigaciones en Eslovenia, Salamanca y Finlandia». Verba Hispanica, 17, 165-182.

Šifrar Kalan, M. (2012): «Análisis comparativo de la disponibilidad léxica en español como lengua extranjera (ELE) y lengua materna (ELM)». MarcoELE. Revista de didáctica español lengua extranjera, 15: https://marcoele. com/disponibilidad-lexica/ (11-11-2019).

Vermunt, J. D. (1994). Inventory of learning styles in bigher education. Maastricht: Maastricht University.

Vermunt, J. D. (1996): «Metacognitive, cognitive and affective aspects of learning styles and strategies: a phenomenographic analysis». Higher Education, 31, 25-50.

Willingham, D. T., Hughes, E. M., Dobolyi, D. G. (2015): «The scientific status of learning styles theories». Teaching of Psychology, 42(3), 266-271. 


\section{Anexo 1. Diapositivas de los centros de interés}

\section{Individuo: dimensión perceptiva y} anímica

- Carácter y personalidad

- Sentimientos y estados de ánimo

- Sensaciones y percepciones físicas

- Estados mentales

- Modales y comportamiento

- Valores personales

- Suerte

3. Salud e higiene

- Enfermedades

- Heridas y traumatismos

- Síntomas

- Centros de asistencia sanitaria

- Medicina y medicamentos

- Estética

5. Geografía y naturaleza

- Universo y espacio

- Espacios urbanos y rústicos

- Clima y tiempo atmosférico

- Fauna

- Flora

- Problemas medioambientales y desastres naturales

\section{Individuo: dimensión física}

- Partes del cuerpo

- Características físicas

- Acciones y posiciones que se realizan con el cuerpo

- Ciclo de vida y reproducción

Anexo 2. Plantillas de respuesta de la prueba de disponibilidad léxica

\begin{tabular}{|l|l|l|}
\hline 1. Individuo: dimensión perceptiva y anímica \\
\hline 1 & 14 & 27 \\
\hline 2 & 15 & 28 \\
\hline 3 & 16 & 29 \\
\hline 4 & 17 & 30 \\
\hline 5 & 18 & 31 \\
\hline 6 & 19 & 32 \\
\hline 7 & 20 & 33 \\
\hline 8 & 21 & 34 \\
\hline 9 & 22 & 35 \\
\hline 10 & 23 & 36 \\
\hline 11 & 24 & 37 \\
\hline 12 & 25 & 38 \\
\hline 13 & 26 & 39 \\
\hline
\end{tabular}


Anexo 3. Índices de disponibilidad léxica individual

\begin{tabular}{|c|c|c|c|c|c|c|c|}
\hline Informantes & $\mathbf{C l} \mathbf{1}$ & $\mathbf{C l} \mathbf{2}$ & $\mathbf{C l} \mathbf{3}$ & $\mathrm{Cl} \mathbf{4}$ & $\mathrm{Cl} \mathbf{5}$ & $\mathrm{Cl} \mathbf{6}$ & TOTAL \\
\hline $\mathbf{1}$ & 0,102 & 0,022 & 0,059 & 0,05 & 0,037 & 0,043 & $\mathbf{0 , 3 1 3}$ \\
\hline $\mathbf{2}$ & 0,13 & 0,021 & 0,008 & 0,038 & 0,035 & 0,094 & $\mathbf{0 , 3 2 6}$ \\
\hline $\mathbf{3}$ & 0,065 & 0,009 & 0,066 & 0,04 & 0,046 & 0,086 & $\mathbf{0 , 3 1 2}$ \\
\hline $\mathbf{4}$ & 0,115 & 0,023 & 0,043 & 0,059 & 0,046 & 0,067 & $\mathbf{0 , 3 5 3}$ \\
\hline $\mathbf{5}$ & 0,07 & 0,03 & 0,03 & 0,06 & 0,063 & 0,108 & $\mathbf{0 , 3 6 1}$ \\
\hline $\mathbf{6}$ & 0,084 & 0,026 & 0,043 & 0,043 & 0,03 & 0,102 & $\mathbf{0 , 3 2 8}$ \\
\hline $\mathbf{7}$ & 0,102 & 0,035 & 0,048 & 0,05 & 0,083 & 0,097 & $\mathbf{0 , 4 1 5}$ \\
\hline $\mathbf{8}$ & 0,081 & 0,012 & 0,063 & 0,039 & 0,075 & 0,076 & $\mathbf{0 , 3 4 6}$ \\
\hline $\mathbf{9}$ & 0,079 & 0,017 & 0,038 & 0,039 & 0,037 & 0,042 & $\mathbf{0 , 2 5 2}$ \\
\hline $\mathbf{1 0}$ & 0,1 & 0,019 & 0,035 & 0,023 & 0,069 & 0,068 & $\mathbf{0 , 3 1 4}$ \\
\hline $\mathbf{1 1}$ & 0,08 & 0,026 & 0,04 & 0,058 & 0,047 & 0,1 & $\mathbf{0 , 3 5 1}$ \\
\hline $\mathbf{1 2}$ & 0,12 & 0,038 & 0,043 & 0,058 & 0,039 & 0,06 & $\mathbf{0 , 3 5 8}$ \\
\hline $\mathbf{1 3}$ & 0,112 & 0,025 & 0,059 & 0,048 & 0,063 & 0,114 & $\mathbf{0 , 4 2 1}$ \\
\hline $\mathbf{1 4}$ & 0,101 & 0,015 & 0,046 & 0,047 & 0,053 & 0,071 & $\mathbf{0 , 3 3 3}$ \\
\hline $\mathbf{1 5}$ & 0,074 & 0,022 & 0,057 & 0,034 & 0,021 & 0,06 & $\mathbf{0 , 2 6 8}$ \\
\hline $\mathbf{1 6}$ & 0,084 & 0,018 & 0,042 & 0,045 & 0,026 & 0,083 & $\mathbf{0 , 2 9 8}$ \\
\hline $\mathbf{1 7}$ & 0,092 & 0,02 & 0,027 & 0,016 & 0,034 & 0,058 & $\mathbf{0 , 2 4 7}$ \\
\hline $\mathbf{1 8}$ & 0,069 & 0,024 & 0,025 & 0,063 & 0,05 & 0,095 & $\mathbf{0 , 3 2 6}$ \\
\hline $\mathbf{1 9}$ & 0,114 & 0,037 & 0,029 & 0,063 & 0,038 & 0,042 & $\mathbf{0 , 3 2 3}$ \\
\hline $\mathbf{2 0}$ & 0,098 & 0,037 & 0,052 & 0,066 & 0,061 & 0,124 & $\mathbf{0 , 4 3 8}$ \\
\hline $\mathbf{2 1}$ & 0,103 & 0,018 & 0,037 & 0,04 & 0,074 & 0,103 & $\mathbf{0 , 3 7 5}$ \\
\hline $\mathbf{2 2}$ & 0,081 & 0,03 & 0,049 & 0,062 & 0,064 & 0,11 & $\mathbf{0 , 3 9 6}$ \\
\hline $\mathbf{2 3}$ & 0,111 & 0,023 & 0,052 & 0,036 & 0,037 & 0,104 & $\mathbf{0 , 3 6 3}$ \\
\hline $\mathbf{2 4}$ & 0,104 & 0,011 & 0,049 & 0,051 & 0,055 & 0,134 & $\mathbf{0 , 4 0 4}$ \\
\hline $\mathbf{2 5}$ & 0,078 & 0,031 & 0,042 & 0,036 & 0,04 & 0,087 & $\mathbf{0 , 3 1 4}$ \\
\hline $\mathbf{2 6}$ & 0,1 & 0,027 & 0,05 & 0,057 & 0,049 & 0,117 & $\mathbf{0 , 4 0 0}$ \\
\hline $\mathbf{2 7}$ & 0,07 & 0,021 & 0,022 & 0,055 & 0,041 & 0,075 & $\mathbf{0 , 2 8 4}$ \\
\hline
\end{tabular}


Javier Domínguez Pelegrín

National Distance Education University (UNED)

\section{The incidence of learning styles in the index of individual lexical availability of foreign Spanish students}

Keywords: learning styles, lexis, lexical availability, available lexicon, Spanish as a foreign language

The concept of learning styles emerged in the field of psychology in the 1930's. Nevertheless, it did not appear in the field of languages teaching until the 1970's due to the study of the good apprentice (Reid, 1975). On the other hand, lexical availability is a branch of study of applied linguistics, which emerged in Latin America at the beginning of the 1970's, whose aim is to develop the atlas of the Spanish available lexicon; that is, provide the lexical units that speakers from each region use more often according to the topic of conversation. In this article, we describe an empirical study in which these two elements, learning styles (LS) and lexical availability (LA), are related and whose fundamental aspects are addressed in the theoretical framework. Next, in the methodology we reveal the objectives and hypotheses of the research, the sample and the instruments. Finally, we present the results of the study. 
Javier Domínguez Pelegrín

Nacionalna univerza za študij na daljavo (UNED)

\section{Vpliv učnih stilov na kazalec individualne leksikalne razpoložljivosti pri tujih učencih španščine}

Ključne besede: učni stili, besedišče, leksikalna razpoložljivost, razpoložljivo besedišče, španščina kot tuji jezik

Koncept učnih stilov se pojavi v psihologiji v 30. letih 20. stoletja, od koder se v 70. letih preko Reidove študije o dobrem učencu (1975) razširi tudi na področje poučevanja jezikov. Leksikalna razpoložljivost pa spada med raziskovalna področja uporabnega jezikoslovja, razvije pa se v Hispanoameriki v začetku 6o. let, in sicer da bi pripomogla k izdelavi atlasa razpoložljivega besedišča v španščini, tj. da bi zbrala leksikalne enote, ki so govorcem posameznih regij najbolj razpoložljive glede na temo pogovora.

V pričujočem prispevku opišemo empirično študijo, v kateri povežemo oba koncepta, učne stile in leksikalno razpoložljivost, njune temeljne vidike pa obdelamo v teoretskem delu. V metodološkem delu nato opredelimo cilje in hipoteze raziskave in opišemo vzorec in uporabljene instrumente, na koncu pa predstavimo rezultate študije. 